\title{
NOTE ON NORMALITY IN QUASI-GROUPS
}

\author{
D. C. MURDOCH
}

An interesting problem in the theory of quasi-groups is to determine how strong an associative law must be assumed in order to obtain a theory of normal subquasi-groups similar to that of ordinary groups. The properties of normal subgroups which it is desirable to retain in the non-associative case are, first, that they form a Dedekind structure, and second, that each one gives rise to a quotient group homomorphic to the whole group. In this note we shall take the latter property as the definition of normality and show that the former follows from it under very general conditions.

We shall understand by a quasi-group $G$ a system of elements satisfying the following two postulates:

I. Product axiom. Any ordered pair of elements $a, b$ of $G$ has $a$ unique product ab which also belongs to $G$.

II. Quotient Axiom. For any two elements $a$ and $b$ of $G$ there exist unique elements $x$ and $y$ of $G$ such that

$$
a x=b, \quad y a=b .
$$

We shall make one further assumption, namely that there exist left coset expansions with respect to any subquasi-group $H$ of $G$. In other words, if $H$ is any subquasi-group, the cosets $a H$ and $b H$ are either identical or have no elements in common. This assumption has been shown by Hausmann and Ore [1] to be equivalent to the following:

III. Weak ASsociative LAW. If $a$ and $b$ are arbitrary elements of $G$ and $c_{0}$ a fixed element, let $d_{0}$ be determined so that $(a b) c_{0}=a d_{0}$. Then for any $c$

$$
(a b) c=a d,
$$

where $d$ belongs to the quasi-group $\left\{c_{0}, d_{0}, c\right\}$ generated by $c_{0}, d_{0}$ and $c$.

Since no confusion can arise, we shall for convenience use the term subgroup for subquasi-group and quotient group for quotient quasigroup, without implying thereby that the systems in question are associative.

It should be noted that a theory of normal subgroups has been given by Hausmann and Ore [1] based on the definition of a normal subgroup as a subgroup $H$ such that $a H=H a$ for all $a$ in $G$. This 
theory requires that $G$ should satisfy associative laws considerably stronger than III in order that the normal subgroups so defined shall have the desired properties. To overcome this difficulty we shall adopt instead the following:

Definition: $A$ subgroup $H$ of $G$ shall be called normal if

$$
(a H)(b H)=(a b) H,
$$

for all elements $a$ and $b$ of $G$.

This is the same definition used in a previous paper by the author [2] where it was applied, however, only to quasi-groups satisfyng rather special associative laws. It will be shown here that the results of that paper concerning normal subgroups can be extended to any quasi-group satisfying III.

Comparing this definition with that of Hausmann and Ore, it is seen that neither one implies the other. Under the latter all subgroups of a commutative quasi-group are normal, while under the former this property is enjoyed by the "abelian" quasi-groups [2] which satisfy the law $(a b)(c d)=(a c)(b d)$. In a Suschkewitsch quasi-group [3] (having unique right unit $e$ and satisfying $a(b c)=(a b)(e c)$, the right unit $e$ is a subgroup which is normal in the sense of this paper but not in the sense of Hausmann and Ore.

Examples of quasi-groups satisfying III but not satisfying the stronger laws previously discussed can easily be constructed by rearranging the elements in the Cayley square of a group, or known quasi-group, so as to destroy the associativity without disturbing the existence of cosets. The following, for example,

\begin{tabular}{l|lllllllll}
\multicolumn{1}{l}{} & 1 & 2 & 3 & 4 & 5 & 6 & 7 & 8 & 9 \\
\hline 1 & 1 & 3 & 2 & 5 & 6 & 4 & 9 & 7 & 8 \\
2 & 3 & 2 & 1 & 6 & 4 & 5 & 7 & 8 & 9 \\
3 & 2 & 1 & 3 & 4 & 5 & 6 & 8 & 9 & 7 \\
4 & 6 & 4 & 5 & 7 & 8 & 9 & 2 & 3 & 1 \\
5 & 4 & 5 & 6 & 8 & 9 & 7 & 3 & 1 & 2 \\
6 & 5 & 6 & 4 & 9 & 7 & 8 & 1 & 2 & 3 \\
7 & 8 & 9 & 7 & 3 & 1 & 2 & 6 & 5 & 4 \\
8 & 9 & 7 & 8 & 1 & 2 & 3 & 4 & 6 & 5 \\
9 & 7 & 8 & 9 & 2 & 3 & 1 & 5 & 4 & 6
\end{tabular}

satisfies III since cosets exist with respect to all subgroups. It is easily verified that it satisfies none of the laws previously discussed by the author nor the laws $A_{2}, A_{3}, A_{4}$ or $A_{5}$ of Hausmann and Ore. 
It is natural to require next that every element of a coset $a H$ shall define the same coset $a H$, or in other words that $(a h) H=a H$ for all $h$ in $H$. In this connection we have the following:

THEOREM 1. The necessary and sufficient condition that every coset aH may be defined by any one of its elements is that $H$ contain the right unit of every element of $G$.

Proof. If $H$ contains all right units of $G$, then $a H=(a h) H$ for all $h$ in $H$, since each coset contains the element $a h$. Conversely if $a H=(a h) H$ for all $h$ in $H$ and all $a$ in $G$, then $a h$ must belong to $(a h) H$. Hence $H$ contains the right unit of $a h$ for all $a$ in $G$ and therefore contains all right units of $G$.

The main object of this note is to prove that in any finite quasigroup the normal subgroups which contain all right units of $G$ form a Dedekind structure. With a slight strengthening of the definition of normality the result may also be proved in the infinite case. We shall assume for the present, however, that $G$ is finite.

THEOREM 2. If $H$ and $K$ are normal subgroups of $G$ and contain all right units of $G$, their crosscut $H \cap K=D$ is also normal.

Proof. From the normality of $H$ and $K$ we have

$$
(a D)(b D) \subseteq(a b) D,
$$

and since $D$ is finite the equality must hold. Moreover $D$ is not void since it contains all right units of $G$.

THEOREM 3. If $H$ and $K$ are normal and contain all right units of $G$, their union $H \cup K$ consists of all products hk where $h$ belongs to $H$ and $k$ to $K$.

The proof of this theorem is similar to that of Theorem 7 of the author's previous paper already cited. The union $H \cup K$ may therefore be written as the product $H K$.

THEOREM 4. If $H$ and $K$ are normal, and contain all right units of $G$, their union is also normal.

Proof. Using the notation $e_{a}$ for the right unit of $a$, we have, from the normality of $H$ and $K$,

$$
\begin{aligned}
{[a(H K)][b(H K)] } & =\left[\left(a e_{a}\right)(H K)\right]\left[\left(b e_{b}\right)(H K)\right] \\
& =[(a H) K][(b H) K]=[(a H)(b H)] K=[(a b) H] K \\
& =\left[(a b) e_{a b}\right][H K]=(a b)(H K),
\end{aligned}
$$

which completes the proof. 
THEOREM 5. The normal subgroups of $G$ which contain all right units of $G$ form a Dedekind structure.

Proof. From Theorems 2 and 4 these subgroups form a structure. It is only necessary, therefore, to show that if $M \supset H$,

$$
M \cap(H \cup K) \subset H \cup(M \cap K) .
$$

This follows as usual since every element of the left-hand side has the form $h k$ and belongs to $M$. Hence $k$ belongs to $M \cap K$ since $h$ belongs to $M$.

In establishing Theorem 5 we have used the finiteness of $G$ in the proofs of Theorems 2, 3 and 4. This can be avoided by strengthening the definition of normality as follows:

A subgroup $H$ shall be called normal in $G$ if for all elements $a, b$ of $G$ and every element $h$ of $H$ we have

$$
(a h)(b H)=(a b) H, \quad(a H)(b h)=(a b) H .
$$

This of course implies the other definition and is equivalent to it in case $G$ is finite. This strengthening of the definition enables us to prove the quotient axioms for the union and crosscut in Theorems 2 and 3. For example, for the union, the equation $x\left(h_{1} k_{1}\right)=h_{2} k_{2}$ has a unique solution of the form $x=h k$. For choosing $h$ so that $h h_{1}=h_{2}$, it follows from (2) that there exists an element $k$ of $K$ such that

$$
(h k)\left(h_{1} k_{1}\right)=h_{2} k_{2} \text {. }
$$

For the proof of Theorem 4 we have as in the finite case

$$
a(H K)=\left(a e_{a}\right)(H K) \subset(a H) K .
$$

But in the infinite case it is necessary to show also that $(a H) K$ $C a(H K)$. This follows from III, applied in the form of Theorem 7, Chapter I, Hausmann and Ore.

From Theorem 5 the usual theorems concerning normal subgroups follow. In particular, the Jordan-Hölder theorem for principal chains, and the Schmidt-Remak decomposition theorems, as proved by Ore [4] for arbitrary Dedekind structures, will all hold. One interesting question arises which has no counterpart in ordinary groups, namely whether the subgroup $R$, generated by the right units of $G$, is necessarily normal. Although there seems to be no obvious reason to believe that this is true, I have no counterexample. If $R$ is not always normal the question arises whether infinite quasi-groups exist in which the Dedekind structure of normal subgroups has no unit element. 


\section{BiBLIOGRAPHY}

1. B. A. Hausmann and O. Ore, Theory of Quasi-groups, American Journal of Mathematics, vol. 59 (1937), pp. 983-1004.

2. D. C. Murdoch, Quasi-groups which satisfy certain generalized associative laws, American Journal of Mathematics, vol. 61 (1939), pp. 509-522.

3. A. Suschkewitsch, On a generalization of the associative law, Transactions of this Society, vol. 31 (1929), pp. 204-214.

4. O. Ore, On the foundation of abstract algebra I and II, Annals of Mathematics, vol. 36 (1935), pp. 406-437, and vol. 37 (1936), pp. 265-292.

YALE UNIVERSITY 